\title{
Optimisation des paramètres du procédé de pliage en tombé de bord par plan d'expériences numérique et méthode des surfaces de réponse
}

\author{
Slim Ben-Elechi ${ }^{1}$, Riadh Bahloul ${ }^{2, a}$ et Alain Potiron ${ }^{2}$ \\ 1 Laboratoire de Mécanique Roberval, Université de Technologie de Compiègne, 60203 Compiègne, France \\ 2 LPMI-ERTGI, ENSAM, 2 boulevard du Ronceray, 49035 Angers, France
}

Reçu le 18 mars 2005, accepté le $1^{\text {er }}$ décembre 2006

\begin{abstract}
Résumé - Le pliage, en tant que procédé, est une opération de mise en forme à froid d'un matériau plat avec ou sans lubrification, obtenue par dépassement de sa limite élastique. Après recul des outils et relâchement des contraintes, il se produit un retour élastique à l'issue duquel persiste une déformation permanente dépendant des paramètres du pliage utilisés. Ce travail constitue une analyse de ce procédé par la méthode des éléments-finis (EF). On se contente dans l'étude de présenter une méthode numérique fiable pour la prédiction de l'effort de pliage et la distribution de champs des contraintes dans la tôle déformée. La fiabilité d'une telle prédiction dépend de plusieurs paramètres tels que le frottement, les propriétés de matériau et de l'endommagement. Le modèle proposé est développé selon un critère élasto-plastique de type von Mises intégrant le mécanisme d'endommagement tel que formulé par Lemaître en 1985. Le comportement du matériau à l'interface tôle/poinçon est modélisé par la loi de frottement de Coulomb. Le modèle étant développé et la maquette numérique construite sur ABAQUS/Standard, le calcul peut être effectué dans tout le domaine de variation des paramètres considérés. L'influence du rayon de la matrice et du jeu tôle-poinçon a été analysée. Les résultats ont été analysés par la méthode des surfaces de réponse (RSM). L'utilisation des surfaces représentatives pour toutes les combinaisons considérées a pour objectif d'optimiser le procédé et d'identifier le paramètre le plus influant sur la qualité finale de la pièce. La technique d'optimisation par les plans d'expériences qui a été adoptée a conduit à minimiser les contraintes de traction et de compression localisées dans les zones les plus endommagées. Les résultats obtenus montrent la robustesse du modèle proposé et sont analysés en détail dans cette étude.
\end{abstract}

Mots clés : Pliage / méthode EF / optimisation / plan d'expériences / surfaces de réponse

\begin{abstract}
Optimisation of process parameters in wiping die bending using numerical design of experiments and response surface method. Bending has significant importance in sheet metal product industry such as automotive security parts. The present work presents a general framework of numerical simulation of wiping die bending processes. A Computer Aided Design (CAD) for bending process using finite element method is presented which consists of metal forming operation characterised by complete material deformation. In this paper, we describe robust methods of predicting load bending evolution and stress distribution of specimens in 3D modelling subjected to bending deformation. The values are determined in a local frame according to elements deformation. A numerical approach of the problem requires a comprehensive finite element modelling due to the diversity of physical phenomena involved, such as friction effects, material and geometrical non-linearities, large plastic deformation and material failure. Lemaître's material damage within the sheet was taken into account in the simulation during forming process. The interface contact law is defined by Coulomb's friction model and the elastoplastic constitutive laws are integrated by means of an incremental formulation which has been implemented in the finite element code ABAQUS. The influence of punch/die clearance and die shoulder radius are analysed. An algorithmic loop programmed in Script language of ABAQUS was retained to investigate the mechanical responses of the part for each case of parameters combination. The punch load and the distribution of stresses could be predicted in view of optimising the value of the main parameters of the process. An optimisation technique based on the use of Design Of Experiments (DOE) and Response Surface Methodology (RSM) is proposed in the work in order to minimise the tensile and compressive stresses found in the most damaged zone during the bending operation. Numerical results have shown the robustness of the proposed model and are discussed in more detail and enlightened by corresponding plots.
\end{abstract}

Key words: Bending / FE method / optimisation / DOE / RSM

\footnotetext{
a Auteur correspondant : bahloul_riadh@yahoo.fr
} 


\section{Introduction}

La fabrication de pièces à partir de tôles est généralement réalisée sur des presses à l'aide d'un poinçon et d'une matrice. Les critères de qualité du produit étant de plus en plus sévères en terme de précision géométrique et de performances mécaniques, l'optimisation du procédé de mise en forme est très souvent rendue nécessaire.

Selon Parniere [1], la réussite d'un produit fabriqué par emboutissage, estampage ou plus particulièrement par pliage, dépend essentiellement de trois facteurs qui sont : (a) les caractéristiques physiques de la tôle, (b) la géométrie des outils (c) la lubrification des outillages.

Ces trois facteurs ne sont pas indépendants dans la mesure où une bonne conception des pièces alliée à une bonne géométrie des outils permet dans certains cas de pallier des propriétés mécaniques médiocres du matériau utilisé.

Dans l'étude qui suit, on s'intéresse à une pièce en tôle destinée à caractériser le comportement mécanique de pièces de sécurité automobile (ferrures d'ancrage) mises en forme par pliage. Deux aspects de cette opération sont abordés qui sont relatifs pour le premier au procédé (pliage par tombé de bord) et pour le second à la pièce (champ de contraintes). Le premier aspect concerne l'outillage et plus particulièrement l'évolution de l'effort maximal de pliage en fonction de certains paramètres du procédé. Le second aspect concerne la distribution des contraintes dans la partie centrale de la pièce au voisinage de l'arrondi de pliage, qui est la zone la plus sollicitée par la mise en forme.

La méthode choisie pour une optimisation du procédé est la méthode des Surfaces de Réponse (M.S.R.) s'appuyant sur un plan d'expériences obtenu par des simulations numériques. Celles-ci découlent du développement d'un modèle de calcul par élément-finis sur le code ABAQUS Standard [2] prenant en compte le couplage élastoplasticité et endommagement [3].

L'optimisation du procédé et ses conséquences sur les propriétés mécaniques des pièces est faite sous forme paramétrique à l'aide d'une boucle algorithmique en langage Script d'ABAQUS. L'évolution de l'effort poinçon nécessaire au pliage et la distribution des contraintes dans les zones les plus sollicitées, ont pu ainsi être prédites afin d'identifier les valeurs optimales des principaux paramètres du procédé.

\section{Objectifs de l'étude}

L'étude numérique du pliage sur des éprouvettes trouées fait suite à l'étude expérimentale [4] et à sa confrontation à quelques résultats tirés des modèles éléments-finis [5]. Si les essais de pliage rendent compte de la réalité, certaines analyses ne sont pas facilement accessibles par l'expérience. Ainsi, la distribution des contraintes à l'intérieur de la pièce ou encore l'endommagement du matériau ne peuvent être facilement mesurés lors d'un essai de mise en forme. Par conséquent, pourvu qu'il soit validé dans quelques situations expérimentales représentatives, le modèle numérique prend toute son importance pour une prédiction approfondie du comportement des pièces et (ou) pour optimiser le procédé.

Les objectifs poursuivis dans cette étude sont de deux ordres :

1. Maximisation de la résistance de la pièce en service, obtenue en minimisant les champs de contraintes résiduelles issues du pliage.

2. Minimisation des sollicitations engendrées dans les outillages tel que l'effort maximal de pliage appliqué par le poinçon sur la pièce.

À ces problèmes d'optimisation sont associées des contraintes technologiques et mécaniques telles que la précision des formes, l'apparition de fissures ou encore le plissement de la tôle dans les parties arrondies. La précision des prédictions numériques concernant ces phénomènes reste cependant à valider par une approche expérimentale. Ces aspects ne sont pas abordés dans l'étude.

Les paramètres de l'étude portent sur la géométrie des outillages. Les variables associées sont relatives au poinçon et à la matrice. Ce sont les rayons d'outils, le jeu résiduel tôle-poinçon et l'angle de pliage qui dépend de la forme caractéristique de la matrice. Au contraire, les paramètres du procédé de mise en forme relative à la lubrification et ses effets sur le frottement pièce-outillage et la course du poinçon restent invariables.

\section{Pliage d'éprouvettes trouées}

\subsection{Modélisation numérique}

Dans cette étude nous considérons l'opération de pliage par tombé de bord, encore appelé pliage en L ou à $90^{\circ}$, d'une éprouvette trouée. La simulation numérique est réalisée en $3 \mathrm{D}$ sur ABAQUS. Le principe de cette opération consiste à déformer une tôle, maintenue fixe entre une matrice et un serre-flan, sous l'action d'un poinçon afin d'obtenir un angle de pliage qui ne peut en aucun cas dépasser une valeur de $90^{\circ}$. Les valeurs numériques des paramètres physiques de la simulation sont reportées dans le tableau 1. Afin de se rapprocher de la réalité expérimentale où les contacts entre le poinçon, la matrice, le serre-flan et la tôle sont lubrifiés par imprégnation au pinceau, on choisira un coefficient de frottement côté poinçon $\mu_{\mathrm{p}}$ relativement faible $[4,6]$. Le coefficient de frottement côté matrice $\mu_{\mathrm{m}}$ et serre-flan est plus élevé car voisin d'un frottement sec. La course optimale imposée au poinçon est de $29 \mathrm{~mm}$. Elle a été identifiée expérimentalement dans des travaux précédents [7] et correspond à un retour élastique minimal.

Puisque son influence sur le comportement du matériau est certaine, le dommage est pris en compte dans la simulation tridimensionnelle de l'opération. Dans le cadre d'un endommagement isotrope, on a choisi la formulation de Lemaître [8] où apparaissent les paramètres 
Tableau 1. Paramètres physiques utilisés pour le matériau et le procédé.

\begin{tabular}{ccccccccccc}
\hline & Matériau & \multicolumn{4}{c}{ Modèle de Lemaître } & \multicolumn{2}{c}{ Frottement } \\
\hline$E(\mathrm{MPa})$ & $\nu$ & $\sigma_{\mathrm{e}}(\mathrm{MPa})$ & $K(\mathrm{MPa})$ & $n$ & $\varepsilon_{\mathrm{D}}$ & $\varepsilon_{\mathrm{R}}$ & $D_{\mathrm{c}}$ & $D_{\mathrm{R}}$ & $\mu_{\mathrm{p}}$ & $\mu_{\mathrm{m}}$ \\
\hline 200000 & 0,28 & 560 & 800 & 0,745 & 0 & 0,36 & 0,21 & 0,99 & 0,09 & 0,15 \\
\hline
\end{tabular}

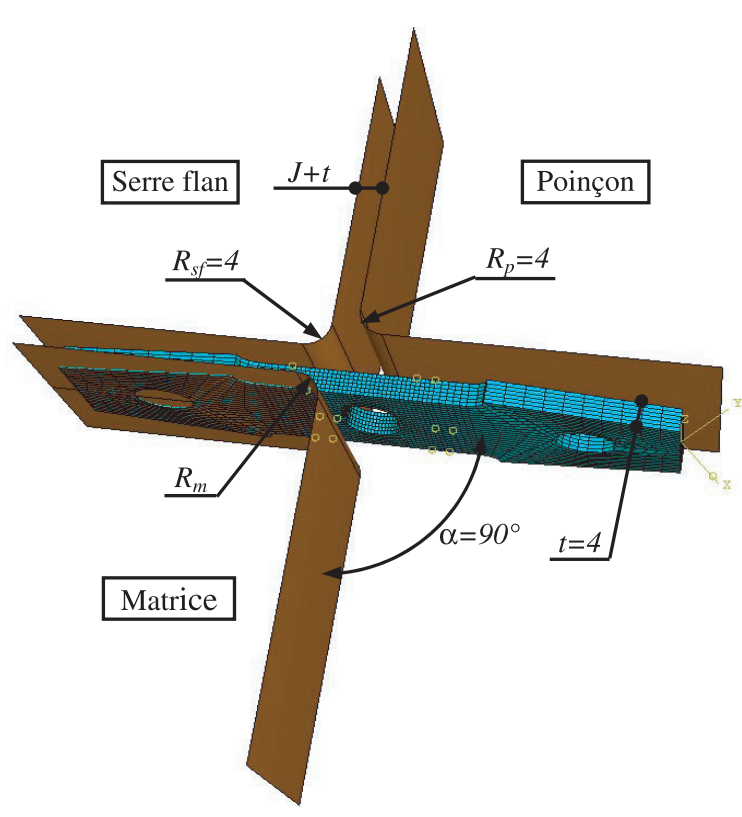

Fig. 1. Maquette numérique du pliage des éprouvettes.

physiques $\varepsilon_{\mathrm{D}}, \varepsilon_{\mathrm{R}}, D_{\mathrm{c}}$ et $D_{\mathrm{R}}$. Ils correspondent respectivement aux déformations plastiques au début de l'endommagement $\left(\varepsilon_{\mathrm{D}}\right)$ et à la rupture $\left(\varepsilon_{\mathrm{R}}\right)$, au dommage critique entraînant la rupture $\left(D_{\mathrm{c}}\right)$ et à la valeur adoptée dans la simulation $\left(D_{\mathrm{R}}\right)$ pour annuler la raideur des éléments-finis totalement endommagés.

La maquette numérique correspondant à l'éprouvette est représentée sur la figure 1 en position initiale dans l'outillage. Dans cette étude, les outils sont modélisés par des corps rigides. Des éléments quadratiques à huit nœuds de type C3D8 (briques à 8 nœuds et 8 points de Gauss) ont été utilisés pour le maillage. Une attention particulière a été portée aux zones adjacentes au trou oblong, car à ce niveau la pièce est pliée et par conséquent les déformations et les niveaux de contraintes y seront importants.

C'est cette maquette numérique, incluant le couplage à l'endommagement, qui est mise en œuvre pour la simulation et la prédiction du comportement des éprouvettes.

Le maillage de la figure 2, montre un raffinement aux abords du trou alors qu'il est plus grossier dans les zones d'attache. Le nombre et la taille moyenne des élémentsfinis notamment dans la zone pliée sont respectivement $0,66 \mathrm{~mm}$ et $3600 \mathrm{~mm}$.

Les états initial et final des éprouvettes durant l'opération de pliage sont montrés par la figure 3. L'investigation numérique proposée de ce procédé de mise en forme doit tenir compte de l'étude de toutes les combinaisons possibles entre le jeu tôle-poinçon variant dans

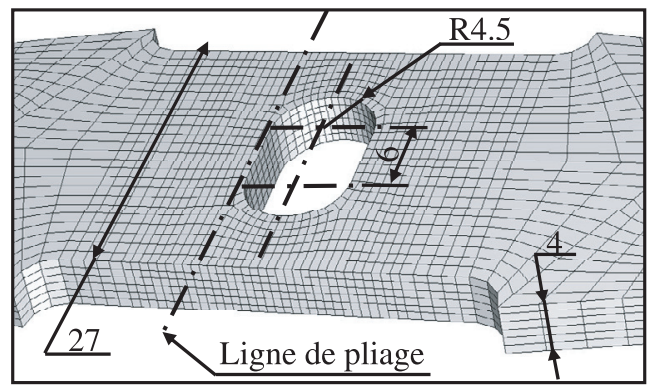

Fig. 2. Maillage E.F dans la zone pliée.

l'intervalle $[-0,6 ; 0,6] \mathrm{mm}$ avec un pas uniforme de $0,2 \mathrm{~mm}$ et le rayon de matrice ayant des valeurs de 1, 2, 4 et $6 \mathrm{~mm}$.

Sachant que la qualité finale de la pièce pliée dépend fortement des paramètres de pliage, de nombreux calculs ont été effectués afin de quantifier leurs effets sur l'écrouissage et l'endommagement du matériau qui sont fonction de la déformation plastique engendrée au pli. Tous les calculs ont été faits avec un ordinateur muni d'un processeur à 2,40 GHz et une mémoire vive de 2096 mégaoctets, chaque calcul prend en moyenne 24 heures.

\subsection{Effort de pliage}

Les simulations numériques ont été réalisées jusqu'à $100 \%$ de la course du poinçon. Différents jeux positifs (Jeu $=0,0,2,0,4$ et $0,6 \mathrm{~mm}$ ) ont été associés à différents rayons de matrice $\left(R_{\mathrm{m}}=1,2,4\right.$ et $\left.6 \mathrm{~mm}\right)$. Les figures 4 et 5 montrent les évolutions de l'effort de pliage relevé sur le poinçon en fonction de la pénétration. Pour un déplacement très faible, l'effort fourni par le poinçon augmente très rapidement jusqu'à atteindre à peu prés $50 \%$ de sa valeur maximale. Ceci correspond essentiellement à l'accroissement des contraintes nécessaires à la plastification de la section d'éprouvette située au voisinage du rayon de la matrice. Au fur et à mesure que le poinçon se déplace, il y a un phénomène d'enroulement de la pièce sur la matrice et la distance entre les points de contact de la tôle avec d'une part le poinçon et d'autre part la matrice diminue. En conséquence, pour un même moment plastique l'effort augmente. On constate aussi qu'il existe à peu près un rapport de 2 entre l'effort nécessaire à la première plastification et l'effort maximum.

Comme on le voit sur les figures 4 et 5 , l'effort est maximal pour un déplacement d'environ $6 \mathrm{~mm}$ correspondant à environ de $20 \%$ du déplacement total du poinçon. Ensuite, il décroît puisque la pièce est en principe pliée à $90^{\circ}$. En effet, il n'est plus nécessaire de fournir un effort de plastification mais le poinçon doit empêcher le retour élastique et vaincre les forces de frottement avec la tôle. 


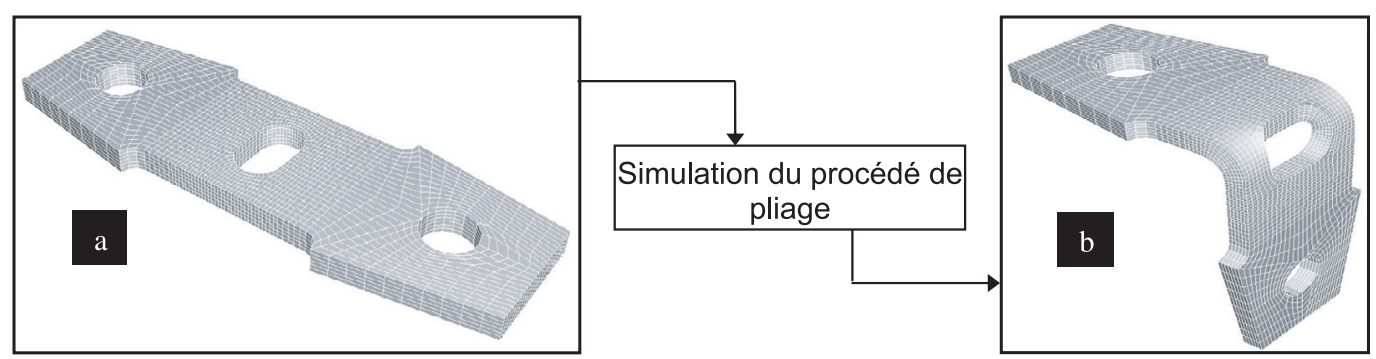

Fig. 3. Géométries initiale (a) et finale (b) de l'éprouvette.

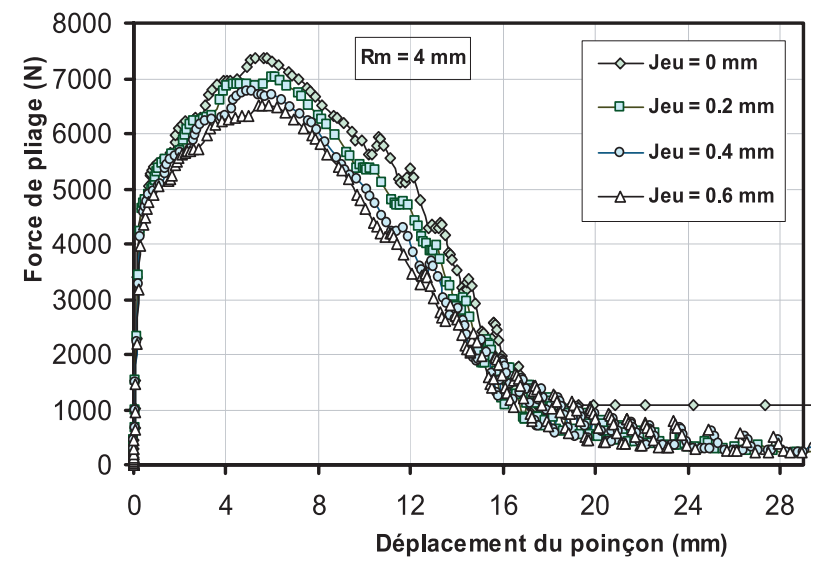

Fig. 4. Courbes force de pliage-déplacement poinçon paramétrées en fonction du jeu pour un $R_{\mathrm{m}}=4 \mathrm{~mm}$.

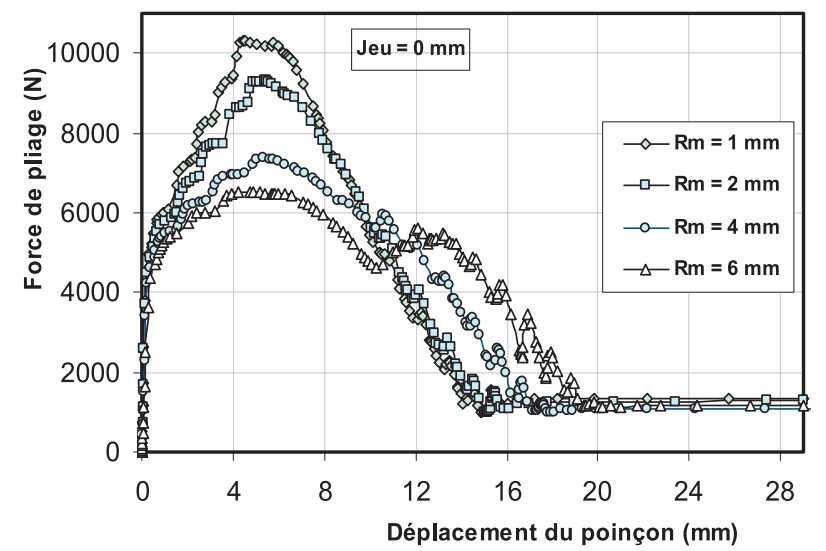

Fig. 5. Courbes force de pliage-déplacement poinçon paramétrées en fonction du rayon de la matrice pour un Jeu $=0 \mathrm{~mm}$.

Comme il s'éloigne de plus en plus de la dernière section pliée, l'effort qu'il doit fournir décroîtra d'une manière sensiblement linéaire. Dans tous les cas, la chute régulière de l'effort conduit en fin de course à une valeur minimale plus ou moins stabilisée qui correspond au frottement entre le poinçon et la tôle.

En théorie [4] pour un jeu nul, la course $\lambda_{\mathrm{p}}$ du poinçon nécessaire au pliage à $90^{\circ}$ devrait être égale à :

$$
\lambda_{\mathrm{p}}=R_{\mathrm{m}}+R_{\mathrm{p}}+t
$$

Pour cette valeur on devrait avoir l'effort maximal. On ne retrouve pas ce résultat sur les figures 4 et 5. L'effort maximal devrait se situer à $\lambda_{\mathrm{p}}=12 \mathrm{~mm}$ pour les combinaisons représentées sur la figure 4 alors qu'il est obtenu à $6 \mathrm{~mm}$.

Pour un rayon de matrice de $4 \mathrm{~mm}$ correspondant au pliage industriel d'une attache de ceinture de sécurité, le jeu $J$ entre la tôle et l'outillage a une influence qui ne dépasse pas $15 \%$ environ entre les valeurs extrêmes 0 et $0,6 \mathrm{~mm}$.

Les courbes de la figure 5 sont assez différentes suivant qu'on plie avec un rayon $R_{\mathrm{m}}$ plus ou moins grand. Comme on pouvait s'y attendre, les efforts maximaux de pliage décroissent notablement lorsque le rayon de matrice croît. Cette diminution de l'effort nécessaire au pliage s'explique par une plastification moins importante des sections pliées avec de grands rayons de matrice. On constate aussi que la valeur $\lambda_{\mathrm{p}}$ croît légèrement avec $R_{\mathrm{m}}$ ce qui est en accord avec la relation (1).

L'observation des courbes des figures 4 et 5 permet de conclure que les paramètres du procédé, jeu tôle-poinçon et rayon de la matrice, influent sur la prédiction des efforts pendant l'opération de pliage, la sensibilité étant plus marquée vis-à-vis du rayon de matrice.

\subsection{Influence du rayon de matrice et du jeu sur la force maximale de pliage $F_{\max }$}

\subsubsection{Influence du rayon de matrice}

On a plus particulièrement étudié l'influence du rayon de matrice $R_{\mathrm{m}}$ sur l'effort maximal de pliage $F_{\max }$ dans le cas où le jeu serait nul, valeur utilisée par les industriels. Les résultats des simulations précédentes sont analysés et ils conduisent au graphe de la figure 6. Ils montrent que l'effort maximal nécessaire au pliage est plus élevé quand le rayon de la matrice décroît et ceci malgré un endommagement plus grand du matériau. On peut justifier ces fortes valeurs par le fait que la sollicitation du matériau étant plus sévère (faibles rayons de matrice), elle engendre une déformation plus importante et un durcissement par écrouissage très prononcé. Le matériau devient en quelque sorte plus résistant et nécessite des efforts plus grands pour le plier.

La différence qui existe entre les valeurs prédites des efforts correspondant aux rayons limites de matrice (1 et $6 \mathrm{~mm})$, laisse présager un dommage plus grand pour les pliages avec de faibles valeurs du rayon de matrice qui nécessitent des efforts de poinçon élevés. 


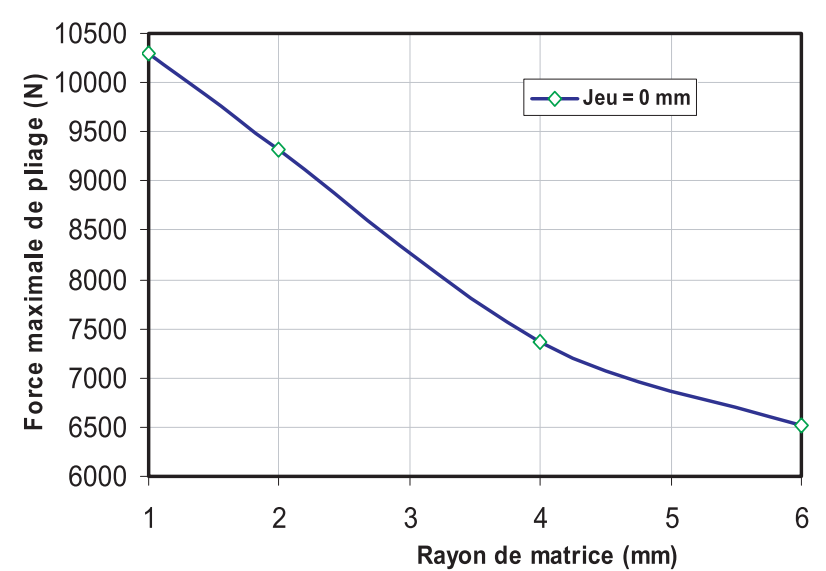

Fig. 6. Variation de la force maximale du pliage $F_{\max }$ en fonction du rayon de la matrice pour un Jeu $=0 \mathrm{~mm}$.

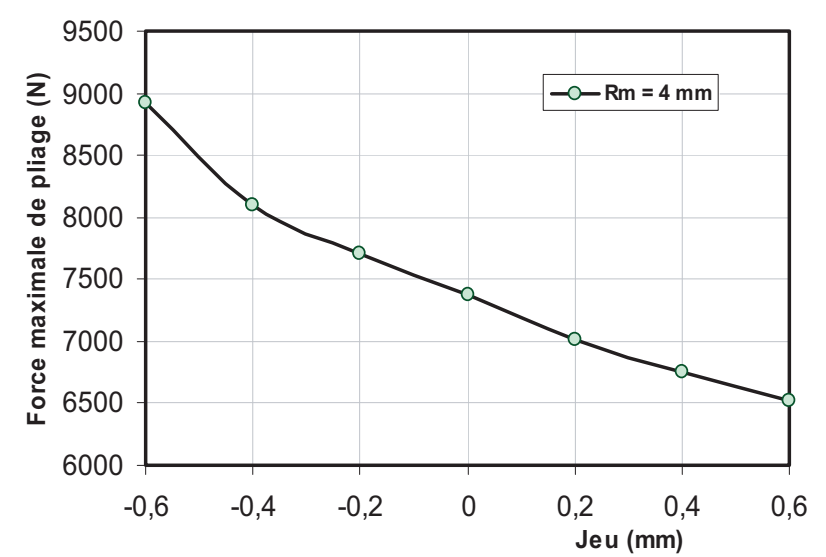

Fig. 7. Variation de la force maximale du pliage $F_{\max }$ en fonction du jeu poinçon/tôle pour un $R_{\mathrm{m}}=4 \mathrm{~mm}$.

\subsubsection{Influence du jeu}

Sur la figure 7 nous avons représenté l'évolution de l'effort maximal obtenu pendant la phase de pliage, en fonction du jeu tôle-poinçon. Les autres paramètres du procédé conservent des valeurs fixes.

Les jeux négatifs sont obtenus lorsqu'on plie des tôles dans un espace poinçon/matrice inférieur à l'épaisseur. Ces jeux sont assez souvent utilisés par les industriels qui cherchent à réduire le retour élastique des pièces pliées.

Nous avons pu constater que la valeur de $F_{\max }$ est d'autant plus importante que le jeu est faible, ce qui semble logique et peut s'expliquer par les effets importants du frottement entre le poinçon et la tôle durant cette phase.

Pour les jeux négatifs, on constate d'après le modèle que nous avons affaire à une sorte de laminage de la tôle ce qui explique l'augmentation de l'effort poinçon, qui de ce fait semble entraîner la partie de tôle en contact. Ce phénomène apparaît un peu avant que l'angle n'atteigne $90^{\circ}$ et se poursuit tout au long de la fin de course. Cette résistance à l'avancement du poinçon se traduit donc par une augmentation de force nécessaire au pliage.
Si on cherche à réduire les sollicitations appliquées sur l'outillage à travers un choix optimal du jeu, la figure 7 suggère de prendre un jeu de $0,6 \mathrm{~mm}$. Malheureusement, ce jeu induira un retour élastique $\theta_{\max }$ plus important et l'optimum sera un compromis à trouver entre $F_{\max }$ et $\theta_{\max }$.

\section{Variation des contraintes $\sigma_{11}$}

\section{1 Évolution dans l'épaisseur (surface externe du trou oblong)}

Des études antérieures [7] sur le pliage d'éprouvettes pleines avaient montré que les contraintes dans la zone pliée se distribuaient en suivant des lignes à peu près parallèles au contour circulaire de l'arrondi. La recherche des contraintes $\sigma_{11}$ dans ces directions peut donc être une aide précieuse afin de mieux comprendre le comportement de l'éprouvette engendré par l'écoulement plastique. Pour des jeux variant dans l'intervalle $[-0,6 \mathrm{~mm}, 0,6 \mathrm{~mm}]$ avec un pas uniforme de $0,2 \mathrm{~mm}$ on a étudié la distribution des contraintes $\sigma_{11}$. Deux cas limites sont envisagés pour les rayons de matrice : 1 et $6 \mathrm{~mm}$. Les valeurs des contraintes sont reportées en fin de pliage et avant l'apparition du retour élastique, c'est-à-dire avant le retrait des outils. Elles représentent la moyenne des valeurs calculées aux points d'intégration et affichées pour chaque élément-fini. Elles sont représentées dans une section où le dommage est maximal en fonction de la position des éléments situés dans l'épaisseur et en partant de la face interne en contact avec la matrice (voir Fig. 8). Les relevés des valeurs sont faits dans un repère local lié aux éléments qui vont se déformer.

Sur les figures 9a et b, les évolutions des contraintes sur le bord extérieur de la pièce sont tracées en fonction du numéro d'élément respectivement pour les valeurs des rayons de matrice $1 \mathrm{~mm}$ et $6 \mathrm{~mm}$. Pour tous les cas de la figure $9 \mathrm{a}\left(R_{\mathrm{m}}=1 \mathrm{~mm}\right)$, on constate que dans le premier élément les contraintes restent négatives ou nulles quel que soit le jeu. Puis au fur et à mesure que le paramètre du procédé $J$ augmente, les contraintes deviennent positives et les courbes tendent à se confondre entre les éléments 2 et 6 . Cette tendance est confirmée sur la figure $9 \mathrm{~b}\left(R_{\mathrm{m}}=6 \mathrm{~mm}\right)$ mais il semble que la valeur plus grande du rayon matrice amène une redistribution des contraintes négatives dans les deux premiers éléments. En même temps le niveau des valeurs maximales en traction et en compression est un peu plus bas. La matière est de ce fait un peu mieux utilisée.

\section{2 Évolution dans l'épaisseur (surface interne du trou oblong)}

Nous examinons maintenant l'évolution des contraintes sur la face interne du trou oblong où la géométrie joue un grand rôle sur la distribution des contraintes. La figure 10 montre la zone étudiée et précise les points de relevé des valeurs. 


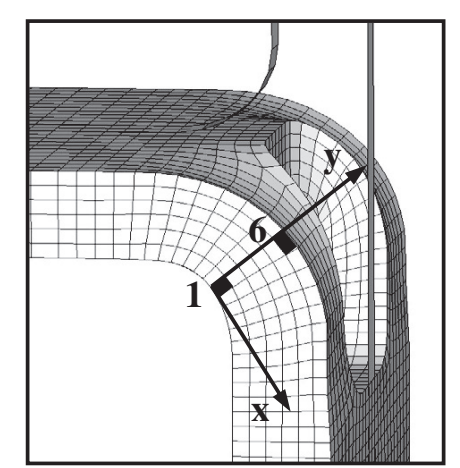

Fig. 8. Géométrie de la zone pliée sur le bord extérieur du pli.
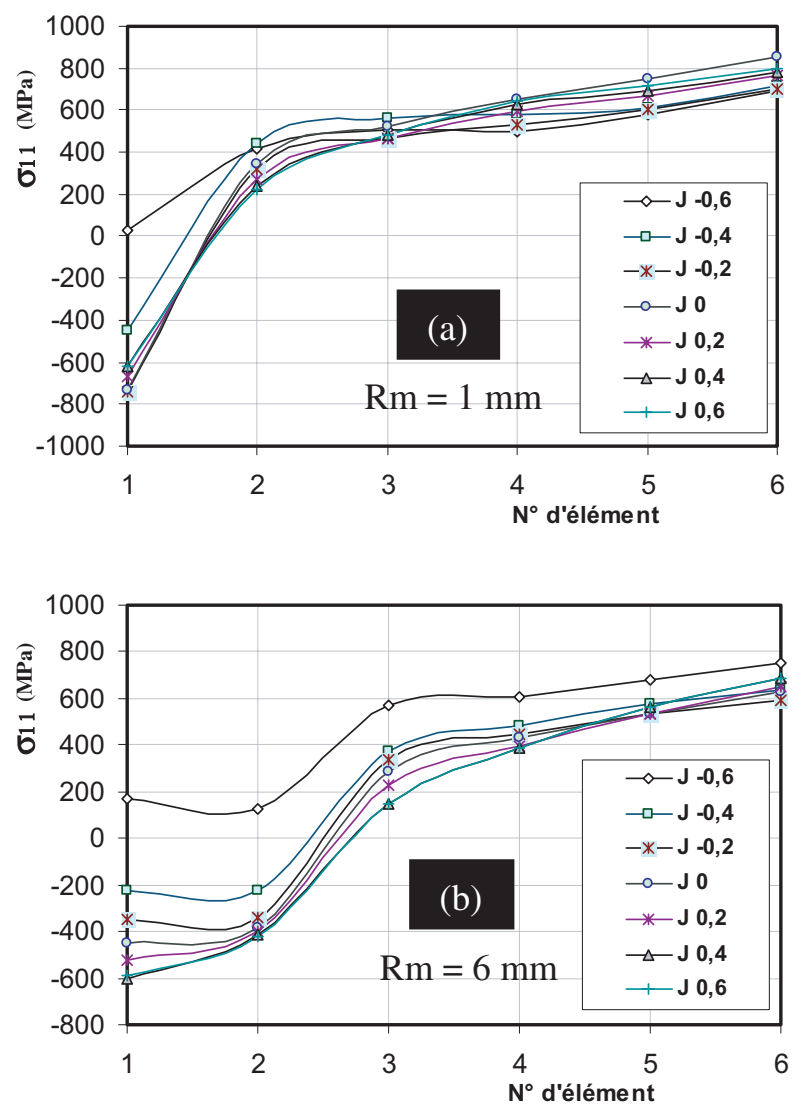

Fig. 9. Évolution de la contrainte $\sigma_{11}$ dans les éléments-finis à travers l'épaisseur de la tôle. Bord extérieur du pli.

Les résultats obtenus (Fig. 11) montrent que :

- La contrainte a une plus faible valeur que précédemment pour le rayon $1 \mathrm{~mm}$ dans la zone de compression de la tôle pour toutes valeurs du jeu tôle-poinçon, bien moins pour le rayon $6 \mathrm{~mm}$. Pour des valeurs négatives du jeu $(J \leqslant-0,4 \mathrm{~mm})$ et un petit rayon de matrice $\left(R_{\mathrm{m}}=1 \mathrm{~mm}\right.$, Fig. 11a), la zone pliée est entièrement en traction. Comme pour la face externe, cette partie en compression apparaît pour des valeurs de $J$ supérieures à $-0,4 \mathrm{~mm}$.

- La figure $11 \mathrm{~b}$ montre que le passage d'un rayon de 1 à $6 \mathrm{~mm}$ n'affecte que les résultats des jeux $J \leqslant-0,4 \mathrm{~mm}$

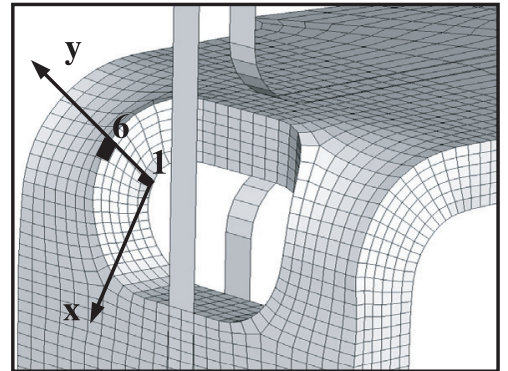

Fig. 10. Zone d'étude de la pièce pliée.
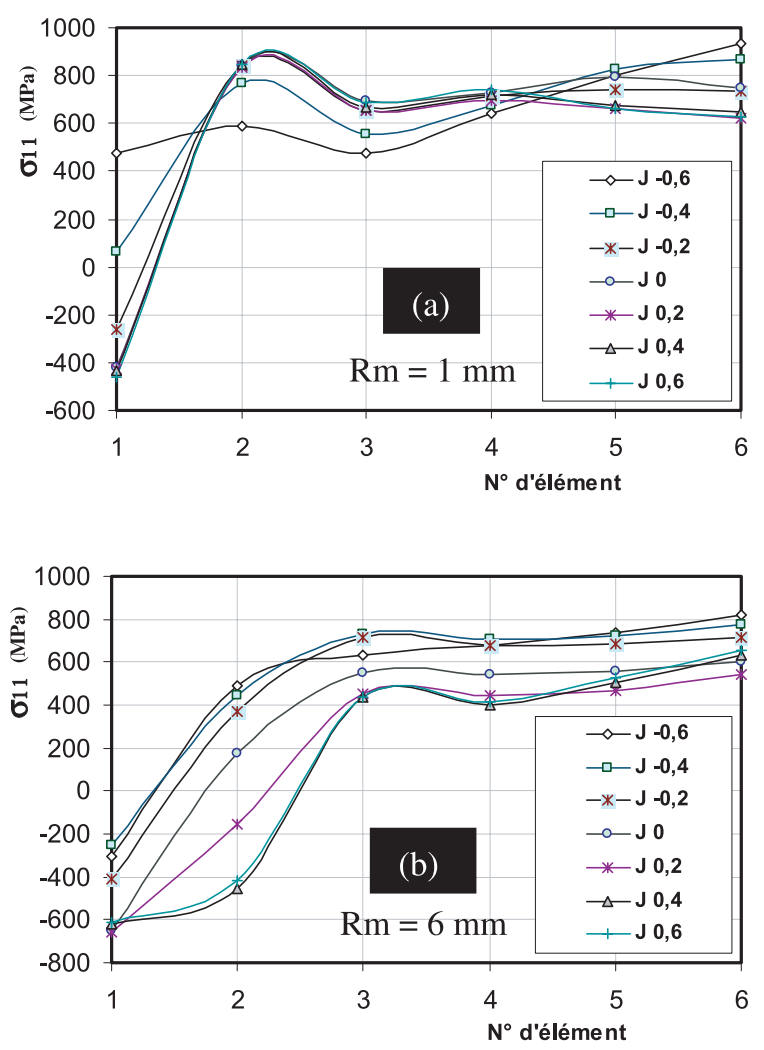

Fig. 11. Évolution de la contrainte $\sigma_{11}$ dans les éléments-finis à travers l'épaisseur de la tôle. Face interne du trou.

mais n'influe pas beaucoup sur le comportement du matériau dans la zone au-dessus de la fibre centrale (éléments 3 à 6 ) où les valeurs sont comprises entre 600 et $800 \mathrm{MPa}$. Pour la zone intérieure (éléments 1 et 2), les jeux négatifs induisent toujours une compression contrairement à ce qui se passe pour $R_{\mathrm{m}}=1 \mathrm{~mm}$ et la différence est surtout marquée dans l'élément 2.

- Pour un rayon de matrice de $1 \mathrm{~mm}$, les zones critiques où la contrainte a une valeur importante sont celles situées environ entre l'élément 2 et 3 , représentant le maxi en compression, et à l'extérieur (élément 6 ) pour la traction. Pour le rayon de $6 \mathrm{~mm}$ la zone critique qui représente le maxi en compression est située sur le bord inférieur du trou oblong. Ceci est essentiellement dû à la dépendance de la position de la fibre neutre par rapport au rayon de pliage. 
Tableau 2. Matrice d'expériences de la force maximale de pliage $(\mathrm{kN})$.

\begin{tabular}{cccccccc}
\hline \multirow{2}{*}{$R_{\mathrm{m}}(\mathrm{mm})$} & \multicolumn{7}{c}{$J(\mathrm{~mm})$} \\
\cline { 2 - 8 } & $-0,6$ & $-0,4$ & $-0,2$ & 0 & 0,2 & 0,4 & 0,6 \\
\hline 1 & 11,831 & 11,650 & 11,081 & 10,303 & 9,954 & 9,373 & 8,828 \\
2 & 11,044 & 10,515 & 9,745 & 9,313 & 8,908 & 8,265 & 7,898 \\
4 & 8,927 & 8,099 & 7,711 & 7,366 & 7,015 & 6,758 & 6,523 \\
6 & 7,239 & 7,035 & 6,843 & 6,514 & 6,422 & 6,523 & 5,989 \\
\hline
\end{tabular}

Tableau 3. Matrice d'expériences de la contrainte $\sigma_{11}(\mathrm{MPa})$ dans l'élément 1 (surface interne du trou).

\begin{tabular}{cccccccc}
\hline \multirow{2}{*}{$R_{\mathrm{m}}(\mathrm{mm})$} & \multicolumn{7}{c}{$J(\mathrm{~mm})$} \\
\cline { 2 - 8 } & $-0,6$ & $-0,4$ & $-0,2$ & 0 & 0,2 & 0,4 & 0,6 \\
\hline 1 & 476,59 & 60,75 & $-263,94$ & $-421,96$ & $-424,5$ & $-431,42$ & $-463,15$ \\
2 & $-22,96$ & $-59,44$ & $-303,65$ & $-559,43$ & $-548,51$ & $-576,39$ & $-586,89$ \\
4 & $-85,43$ & $-77,36$ & $-417,87$ & $-648,54$ & $-658,79$ & $-656,81$ & $-657,05$ \\
6 & $-302,99$ & $-255,88$ & $-411,23$ & $-652,79$ & $-660,63$ & $-623,5$ & $-611,15$ \\
\hline
\end{tabular}

Tableau 4. Matrice d'expériences de la contrainte $\sigma_{11}(\mathrm{MPa})$ dans l'élément 6 (surface interne du trou).

\begin{tabular}{cccccccc}
\hline \multirow{2}{*}{$R_{\mathrm{m}}(\mathrm{mm})$} & \multicolumn{7}{c}{$J(\mathrm{~mm})$} \\
\cline { 2 - 8 } & $-0,6$ & $-0,4$ & $-0,2$ & 0 & 0,2 & 0,4 & 0,6 \\
\hline 1 & 930,41 & 868,36 & 737,71 & 749,17 & 624,32 & 649,62 & 625,99 \\
2 & 852,63 & 847,61 & 773,01 & 772,87 & 709,14 & 723,83 & 742,40 \\
4 & 862,41 & 873,17 & 779,70 & 693,01 & 627,98 & 648,13 & 670,76 \\
6 & 821,89 & 774,62 & 717,60 & 605,20 & 542,48 & 634,56 & 657,64 \\
\hline
\end{tabular}

- La variation du jeu semble modifier aussi la réponse mécanique du matériau et il est intéressant de noter que l'effet du jeu tôle-poinçon est plus prononcé quand le rayon de matrice augmente.

- Compte-tenu des remarques précédentes, si on désire limiter les contraintes en pliage il est conseillé de choisir des valeurs de jeux positives.

\section{Optimisation du procédé}

L'étude numérique de la géométrie des attaches et l'optimisation de leur forme en vue de mieux utiliser la matière ont déjà été présentées [9]. On désire maintenant aborder le problème sous un aspect plus industriel, celui concernant l'optimisation du procédé de pliage par tombé de bord. La démarche retenue utilise la technique des plans d'expériences provenant d'une série de simulations numériques et une approche par surface de réponse. L'étude purement numérique dans ce contexte doit permettre de déterminer les paramètres optimaux du procédé appliqué à la mise en forme de ces éprouvettes en vue de minimiser les forces sur l'outillage et les contraintes maximales générées dans la pièce. Les éprouvettes trouées sont à la base de l'étude.

\subsection{Plans d'expériences}

Nous avons souligné plusieurs fois que la mise en forme des métaux, et le pliage en tombé de bord en particulier, est une opération qui met en œuvre de multiples paramètres. Ici, nous avons restreint l'étude en ne considérant que l'influence des paramètres géométriques les plus significatifs du procédé qui sont le rayon de matrice $R_{\mathrm{m}}$ et le jeu $J$ entre la tôle et le poinçon. Dans la suite de cette étude, la tôle a une épaisseur de $4 \mathrm{~mm}$ et le poinçon un rayon de $4 \mathrm{~mm}$.

L'utilisation des plans d'expériences $[9,10]$ possède l'avantage de structurer les campagnes numérique et expérimentale afin de minimiser le nombre d'essais à réaliser. Nous avons choisi cette technique car dans notre étude il est très difficile d'établir des relations analytiques pour modéliser les différentes réponses mécaniques des pièces au pliage, incluant les paramètres évoqués.

Les fonctions caractéristiques qui ont été choisies pour constituer les réponses des pièces aux sollicitations de pliage, sont l'effort maximal et la contrainte $\sigma_{11}$ dans les zones les plus sollicitées. Ces fonctions sont les fonctions objectives du problème d'optimisation que nous étudierons plus loin. L'effort maximal $F_{\max }^{\text {pliage }}$ est celui qui est prédit par la simulation numérique pendant la phase de pliage et la contrainte $\sigma_{11}$ est celle calculée dans les éléments 1 et 6 de la surface interne du trou (Fig. 10).

Un plan factoriel complet de deux facteurs est utilisé en faisant varier les paramètres de conception, jeu et rayon matrice. Les réponses sont ensuite analysées.

Les tableaux 2-4 récapitulent les résultats du plan d'expériences obtenus avec les intervalles de variation des paramètres $R_{\mathrm{m}}$ et $J$ déjà présentés à la section 3.1 .

\subsection{Surfaces de réponse}

Dans la confection des matrices d'expériences, les variables physiques sont normalisées, c'est-à-dire 
représentées par des valeurs adimensionnelles, de façon à comparer leurs influences respectives sur les réponses, et pouvoir généraliser la méthode à tout type de variables.

Pour cela la force maximale de pliage $F_{\max }^{\text {pliage }}$ est rapportée à la valeur de l'effort élastique $F_{\mathrm{e}}\left(F_{\mathrm{e}}=\mathcal{A} \sigma_{\mathrm{e}}, \mathcal{A}\right.$ est l'aire de la section résistante initiale) et la contrainte $\sigma_{11}$ est normalisée par rapport à la limite élastique $\sigma_{\mathrm{e}}$.

On pose :

$$
\begin{aligned}
& \bar{F}=F_{\max }^{\text {pliage }} / F_{\mathrm{e}} \\
& \bar{\sigma}=\sigma_{11} / \sigma_{\mathrm{e}}
\end{aligned}
$$

Les deux variables retenues pour cette étude, $R_{\mathrm{m}}$ et $J$, sont rapportées à l'épaisseur $t$ de la tôle de sorte que les variables réduites $\bar{J}$ et $\bar{R}_{\mathrm{m}}$ sont définies par :

$$
\bar{J}=J / t \quad \text { et } \quad \bar{R}_{\mathrm{m}}=R_{\mathrm{m}} / t
$$

\subsubsection{Choix d'une forme de solution}

La méthode des surfaces de réponse $[11,12]$ est mise en ouvre afin d'obtenir une approximation des fonctions $\bar{F}$ et $\bar{\sigma}$ à l'aide des variables réduites $\bar{J}$ et $\bar{R}_{\mathrm{m}}$ utilisées. En règle générale on écrit la fonction approchée comme suit :

$$
f=F\left(x_{1}, x_{2}, x_{3}, \ldots, x_{n}\right)+\varepsilon
$$

expression dans laquelle $f$ représente la réponse en fonction des variables $x_{i}(i=1, \ldots, n)$ (paramètres de l'opération) et $\varepsilon$ est un terme représentant l'erreur par rapport à la solution exacte. Dans ce travail, des approximations quadratique et cubique ont été testées et qui ne permettent pas de bien représenter les fonctions objectives. Pour cela, nous avons choisi un polynôme d'ordre quatre avec une interaction entre les variables, ce qui donne :

$$
\begin{aligned}
f & =\alpha_{0}+\sum_{i=1}^{n} \alpha_{i} x_{i}+\sum_{i=1}^{n} \alpha_{i i} x_{i}^{2}+\sum_{i<j}^{n} \alpha_{i j} x_{i} x_{j}+\sum_{i=1}^{n} \beta_{i i} x_{i}^{3} \\
& +\sum_{i=1, i \neq j}^{n} \beta_{i j} x_{i}^{2} x_{j}+\sum_{i=1}^{n} \gamma_{i i} x_{i}^{4}+\sum_{i=1, i \neq j}^{n} \gamma_{i j} x_{i}^{3} x_{j}+\prod_{i=1}^{n} \xi x_{i}^{2}
\end{aligned}
$$

\subsubsection{Optimisation}

L'optimisation est effectuée en vue de rechercher les valeurs optimales des paramètres géométriques du procédé, garantissant une minimisation de l'effort de pliage $F_{\max }^{\text {pliage }}$ et de la contrainte $\sigma_{11}$ localisée dans la zone la plus endommagée de la pièce. Le problème multiobjectif se pose comme suit :

$$
\begin{aligned}
& \text { Minimiser } F_{\max }^{\text {pliage }}=f_{1}\left(R_{\mathrm{m}}, J\right) \\
& \text { Minimiser } \sigma_{11}=f_{2}\left(R_{\mathrm{m}}, J\right)
\end{aligned}
$$

avec les contraintes géométriques :

$$
\begin{aligned}
& R_{\mathrm{m}_{\min }} \leqslant R_{\mathrm{m}} \leqslant R_{\mathrm{m}_{\max }} \\
& J_{\min } \leqslant J \leqslant J_{\max }
\end{aligned}
$$

Les coefficients des polynômes représentant les fonctions approchées $f_{1}$ et $f_{2}$, sont déterminées en minimisant l'erreur quadratique définie par :

$$
\varepsilon_{\mathrm{rr}}=\sqrt{\sum_{i=1}^{n_{\exp }}\left(f-f_{i}\right)^{2}}
$$

expression dans laquelle les $f_{i}$ sont les $n_{\exp }$ valeurs expérimentales que la fonction $f$ devra approcher au mieux. Dans le cas présent, la méthode conduit à résoudre un système linéaire en $\alpha, \beta, \gamma$, et $\xi$ (relation (6), en remplaçant par la forme exacte vous évitez les problèmes sur les coefficients) pour chaque fonction objective $\bar{F}, \bar{\sigma}_{1}$ et $\bar{\sigma}_{6}$. Les fonctions $\bar{\sigma}_{1}$ et $\bar{\sigma}_{6}$ correspondent aux valeurs prises $\sigma_{11}$ dans les éléments 1 et 6 situés dans la face interne du trou oblong (Fig. 10).

Les tableaux 5-7 résument les valeurs des coefficients trouvées pour les trois fonctions objectives.

\subsection{Résultats et analyse}

Les trois fonctions polynomiales déterminées dans la section précédente, permettent de calculer les valeurs des réponses en n'importe quel point du domaine de variation des paramètres du jeu et du rayon grâce à une campagne d'essais numériques donnant les valeurs des $f_{i}$ (relation (9)).

\subsection{1 Étude de l'effort exercé par le poinçon}

Les résultats concernant l'évolution de l'effort maximal $\bar{F}$ au cours du pliage, sont reportés sur les figures 12 a et b. Elles permettent de juger des effets d'une variation des paramètres géométriques du procédé sur la valeur de la charge maximale appliquée par le poinçon pour plier la tôle. L'effet maximum est obtenu pour les faibles valeurs de jeu et de rayon de matrice. La combinaison des paramètres correspondant au maximum global de la force $F_{\max }^{\text {pliage }}$ est obtenue pour $R_{\mathrm{m}}=1 \mathrm{~mm}$ et un jeu égal à $-15 \%$ de l'épaisseur de l'éprouvette. Dans ce cas, la valeur de la fonction objective $F_{\max }^{\text {pliage }}$ atteint $44 \%$ de la force élastique de notre matériau.

Sur la figure $12 \mathrm{a}$, on observe une chute de la force maximale de pliage avec le jeu $J$ tôle/poinçon d'autant plus forte que le rayon de matrice $R_{\mathrm{m}}$ est petit. À jeu égal, on constate aussi que $F_{\max }^{\text {pliage }}$ décroît lorsque $R_{\mathrm{m}}$ croît, ce que nous avions déjà constaté sur la figure 6 . En examinant l'allure de la surface de réponse figure $12 \mathrm{~b}$, on voit qu'il est important d'augmenter le rayon de pliage dans la conception des pièces de sécurité si on désire réduire les efforts engendrés sur les outillages. Le gradient $\frac{\partial F_{\max }^{\text {pliage }}}{\partial R_{\mathrm{m}}}$ 
Tableau 5. Détermination des coefficients de la fonction objective de l'effort relatif de pliage.

\begin{tabular}{ccccccccc}
\hline Coefficients & $\alpha_{0}$ & $\alpha_{1}$ & $\alpha_{2}$ & $\alpha_{11}$ & $\alpha_{22}$ & $\alpha_{12}$ & $\beta_{11}$ & $\beta_{22}$ \\
Valeurs & 0,431 & $-0,161$ & $-0,375$ & $-0,045$ & $-0,369$ & $-0,333$ & 0,047 & 2,883 \\
\hline Coefficients & $\beta_{12}$ & $\beta_{21}$ & $\gamma_{11}$ & $\gamma_{22}$ & $\gamma_{12}$ & $\gamma_{21}$ & $\xi$ & \\
Valeurs & 0,649 & 3,297 & 0,00016 & $-22,628$ & $-0,194$ & $-4,421$ & $-1,767$ & \\
\hline
\end{tabular}

Tableau 6. Détermination des coefficients de la fonction objective $\bar{\sigma}_{1}$ dans l'élément 1 sur la face interne du trou.

\begin{tabular}{ccccccccc}
\hline Coefficients & $\alpha_{0}$ & $\alpha_{1}$ & $\alpha_{2}$ & $\alpha_{11}$ & $\alpha_{22}$ & $\alpha_{12}$ & $\beta_{11}$ & $\beta_{22}$ \\
Valeurs & $-0,393$ & $-1,668$ & $-6,917$ & 0,530 & 75,127 & 15,290 & 1,097 & $-78,940$ \\
\hline Coefficients & $\beta_{12}$ & $\beta_{21}$ & $\gamma_{11}$ & $\gamma_{22}$ & $\gamma_{12}$ & $\gamma_{21}$ & $\xi$ & \\
Valeurs & $-22,219$ & $-38,743$ & $-0,616$ & $-1570,401$ & 8,554 & 154,358 & 14,506 & \\
\hline
\end{tabular}

Tableau 7. Détermination des coefficients de la fonction objective $\bar{\sigma}_{6}$ dans l'élément 6 sur la face interne du trou.

\begin{tabular}{ccccccccc}
\hline Coefficients & $\alpha_{0}$ & $\alpha_{1}$ & $\alpha_{2}$ & $\alpha_{11}$ & $\alpha_{22}$ & $\alpha_{12}$ & $\beta_{11}$ & $\beta_{22}$ \\
Valeurs & 1,105 & 0,665 & $-4,939$ & $-0,303$ & 15,66 & 15,55 & $-0,479$ & 9,253 \\
\hline Coefficients & $\beta_{12}$ & $\beta_{21}$ & $\gamma_{11}$ & $\gamma_{22}$ & $\gamma_{12}$ & $\gamma_{21}$ & $\xi$ & \\
Valeurs & $-20,510$ & $-11,029$ & 0,254 & $-328,308$ & 7,485 & 42,476 & 8,467 & \\
\hline
\end{tabular}
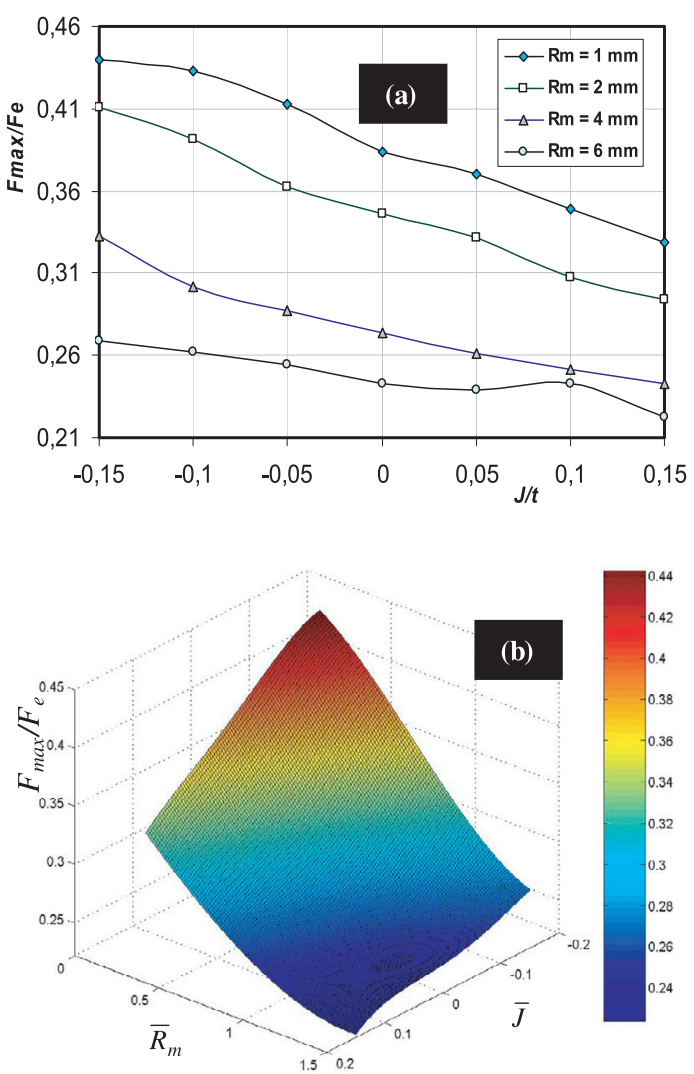

Fig. 12. Évolution de la force maximale relative obtenue en pliage en fonction du jeu relatif pour différents rayons de la matrice (a) et la surface de réponse correspondante (b).

est visiblement plus grand que le gradient $\frac{\partial F_{\max }^{\text {pliage }}}{\partial J}$, une variation de $R_{\mathrm{m}}$ conduira à une plus forte variation de l'effort maximal appliqué par le poinçon, effort nécessaire à la déformation de la tôle.
La valeur minimale de la fonction objective $F_{\text {max }}^{\text {pliage }}$ est de l'ordre de 22,39\% de l'effort élastique, c'est-àdire la moitié de la plus grande charge de la figure 12a. Cette valeur optimale représente le minimum global de la réponse déduite pour les paramètres géométriques du procédé caractérisés par des grandes valeurs $\left(\bar{R}_{\mathrm{m}}=1,46\right.$ et $\bar{J}=0,15)$.

\subsection{2 Étude de la contrainte $\sigma_{11}$}

On s'intéresse maintenant à l'analyse de l'état de contrainte induit par le pliage, en fonction de la variation des paramètres. Les figures $13 \mathrm{a}$ et b montrent la distribution de la contrainte $\sigma_{11}\left(\bar{\sigma}_{1}\right)$ dans l'élément 1 représentée sous forme paramétrique (a) et la surface de réponse associée (b). Dans ce cas, les valeurs de $\sigma_{11}$ sont relevées dans la zone critique la plus endommagée sur la surface interne du trou oblong (Fig. 10). Elles sont relatives à un repère lié aux éléments qui se déplacent.

Mis à part le cas $R_{\mathrm{m}}=1 \mathrm{~mm}$, le champ de contrainte est principalement de type compressif pour toutes les valeurs du jeu relatif $\bar{J}$ supérieures à $-0,1$. Les évolutions de $\sigma_{11}$ ne sont pas uniformes : $\sigma_{11}$ décroît pour des jeux négatifs de plus en plus faibles et se stabilise à partir d'un jeu nul. Dans la zone $(0<\bar{J}<0,15)$ seul le rayon de matrice a une influence sur la valeur de la contrainte maximale $\sigma_{11}$. Dans tout le domaine de variation de $R_{\mathrm{m}}$, on peut voir que les contraintes diminuent lorsqu'on augmente le rayon de pliage.

La surface de réponse associée à la contrainte maximale est tracée sur la figure 13b. En traction, le maximum de la contrainte dans le domaine d'étude, est de l'ordre de 85,11 \% de la valeur de la limite élastique. Il apparaît pour des valeurs relatives du jeu et du rayon de matrice respectivement égales à $-0,15$ et 0,25 . Il n'y a donc pas de 

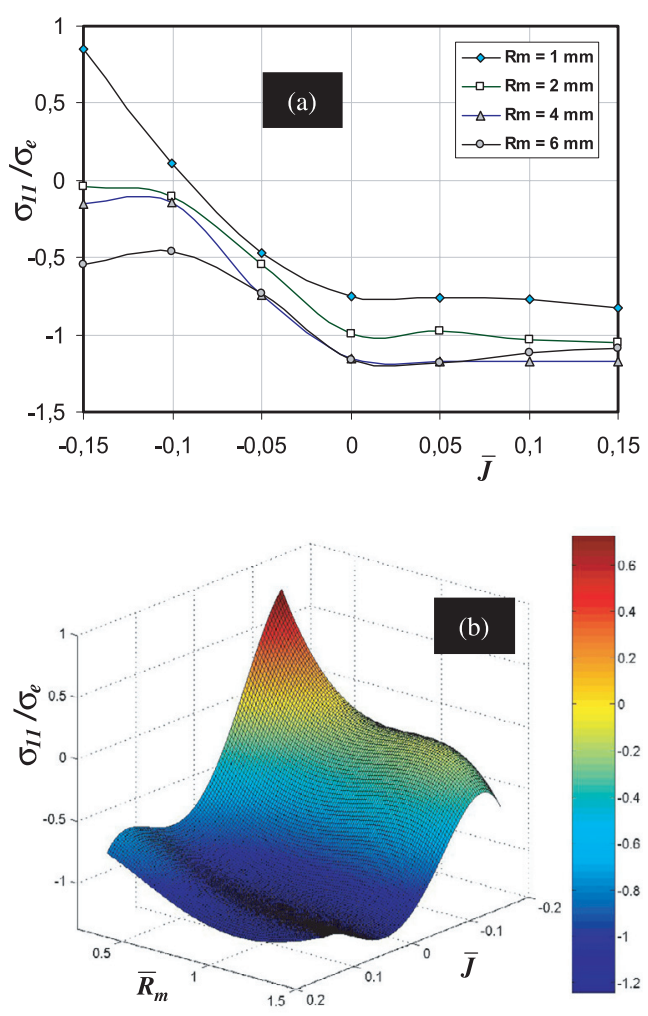

Fig. 13. Évolution de la contrainte relative $\bar{\sigma}_{1}$ dans l'élément 1 en fonction du jeu relatif pour différents rayons de la matrice (a) et la surface de réponse correspondante (b).

plastification. Par contre, la contrainte maximale de compression en valeur absolue dépasse la limite élastique du matériau. Sa valeur est de $1,24 \sigma_{\mathrm{e}}$ et elle est obtenue pour $\bar{J}=0,048$ et $\bar{R}_{\mathrm{m}}=1,5$. Dans ce cas il y a plastification dans l'élément fini étudié (élément 1).

D'une façon similaire, on étudie la variation de la contrainte $\sigma_{11}$ dans l'élément $6\left(\bar{\sigma}_{6}\right)$. Le comportement du matériau dans cet élément, en réponse aux effets des variations des paramètres du procédé, est représenté sous forme d'une surface de réponse visible sur la figure 14b.

La représentation des contraintes sur la figure 14a, montre qu'elles sont toujours positives. Quel que soit le jeu choisi, l'extérieur du pli est en traction. Les contraintes diminuent quand le jeu relatif augmente, avec des courbes d'évolution plus ou moins linéaires suivant la valeur du rayon de matrice. Ceci s'explique par la décroissance de l'écrouissage dans la zone extérieure de la section qui sera pliée avec moins d'intensité.

La surface de réponse donnée par la figure 14b est plus tourmentée que la précédente $\left(\bar{\sigma}_{1}\right.$ Fig. $\left.13 \mathrm{~b}\right)$. Elle présente un minimum global qui correspond à la valeur minimale de la contrainte et un maximum local. Les valeurs de la fonction objective sont respectivement $1,003 \sigma_{\mathrm{e}}$ et $1,32 \sigma_{\mathrm{e}}$, obtenues avec des valeurs de paramètres du procédé correspondant à ces deux extremums qui sont les suivantes : $\left(\bar{R}_{\mathrm{m}}=1,4, \bar{J}=0,069\right)$ et $\left(\bar{R}_{\mathrm{m}}=0,5, \bar{J}=0,15\right)$.

Les résultats numériques mettent en lumière la nonlinéarité de la distribution de contrainte $\left(\sigma_{11} / \sigma_{\mathrm{e}}\right)$ dans
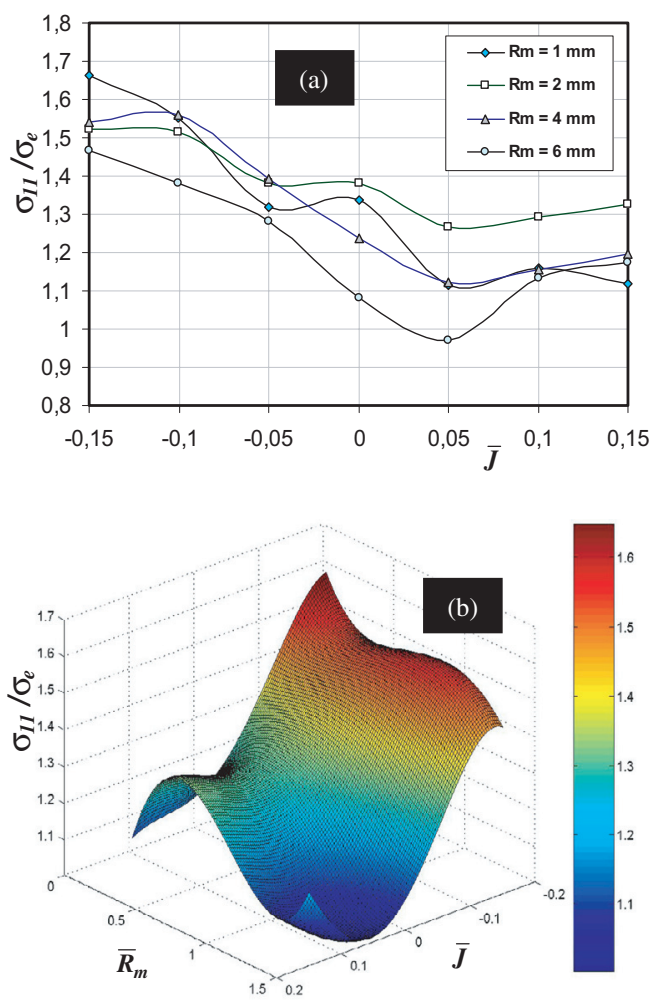

Fig. 14. Évolution de la contrainte relative $\bar{\sigma}_{6}$ dans l'élément 6 en fonction du jeu relatif pour différents rayons de matrice (a) et la surface de réponse correspondante (b).

l'élément 6 avec le rayon de matrice, la valeur maximale de cette réponse étant atteinte pour les plus faibles valeurs des paramètres $\left(\bar{R}_{\mathrm{m}}=0,25, \bar{J}=-0,15\right)$. Elle est approximativement de $1,66 \sigma_{\mathrm{e}}$.

\section{Conclusion}

La mise en forme par pliage à $90^{\circ}$ de pièces trouées a été modélisée en utilisant des éprouvettes comportant un trou oblong, représentatives de ferrures d'ancrage. Cette opération a été étudiée numériquement en vue d'optimiser les paramètres de procédé afin de minimiser l'effort sur le poinçon ou/et les contraintes induites dans la pièce en fin d'opération. Deux paramètres liés au procédé ont été retenus, ils interviennent sous forme adimensionnelle : le rayon matrice réduit $R_{\mathrm{m}} / t$ et le jeu tôle-poinçon réduit $J / t$. On a montré que c'est principalement le rayon de matrice qui est le paramètre prépondérant pour limiter les niveaux d'efforts et de contraintes. Des jeux tôle-poinçon négatifs augmentent les valeurs des réponses en effort et en contrainte. Pour réaliser ces analyses, des plans d'expériences par une approche numérique ont été développés par une modélisation éléments-finis. Les résultats de ces études ont permis d'optimiser le procédé grâce à la mise en place de surfaces de réponse à deux paramètres. Cette technique d'approximation est bien adaptée aux problèmes de mise en forme qui ne peuvent être formalisés analytiquement. 


\section{Références}

[1] P. Parniere, Appréciation des caractéristiques d'emboutissabilité des tôles minces, Mise en forme des métaux et alliages, École d'été de métallurgie physique de Villarssur-Ollon, Suisse, septembre, ISBN2-222-01917-6, 1975, pp. 305-330

[2] Hibbit, Karlsson and Sorensen, Inc, Contact and interaction analysis, ABAQUS/Standard user's manual, volume III, USA, version 6.3, pp. 21.1.1-1 21.3.4-12, 2002

[3] R. Hambli, Étude expérimentale, numérique et théorique du découpage des tôles en vue de l'optimisation du procédé, Thèse de doctorat ENSAM Angers, 1996, pp. $110-171$

[4] R. Bahloul, Optimisation du procédé de pliage sur presses de pièces en tôles à haute limite d'élasticité, Thèse de doctorat, École Nationale Supérieure d'Arts et Métiers d'Angers, 2005

[5] R. Bahloul, A. Mkaddem, Ph. Dal Santo, A. Potiron, Sheet metal bending optimisation using response surface method, numerical simulation and design of experiments, Int. J. Mech. Sci. 48 (2006) 991-1003

[6] C. Magny, Lois de frottement évolutives destinées à la simulation numérique de l'emboutissage, La revue de Métallurgie, CIT/Science et Génie des Matériaux (2002) $145-156$
[7] A. Mkaddem, Expérimentation et simulation du pliage H.L.E. Prévision du comportement en service des pièces pliées, Thèse de doctorat, ENSAM, Angers, 2003

[8] Y. Hammi, Simulation numérique de l'endommagement dans les procédés de mise en forme, Thèse de doctorat, Université de Technologie de Compiègne-Université de Technologie de Troyes, 2000, pp. 179-222

[9] R. Bahloul, P. Dal Santo, A. Potiron, Shape Optimisation of Automotive Security-parts Regarding the Influence of Residual Stresses and Material Damage, 4th European Congress on Computational Methods in Applied Sciences and Engineering, Finland, 2004, p. 490

[10] R. Hambli, A. Potiron, A. Kobi, Application of design of experiment technique for metal blanking processes optimization, Mécanique \& Industries 4 (2003) $175-180$

[11] T. Ohata, Y. Nakamura, T. Katayama, E. Nakamachi, Development of optimum process design system for sheet fabrication using response surface method, J. Materials Processing Technology 143-144 (2003) 667-672

[12] S. Ben Elechi, Analyse et conception rapides de paramètres du procédé d'emboutissage pour le contrôle du retour élastique, Thèse de doctorat, Université de Technologie de Compiègne, 2004, pp. 123-145

Retrouvez nos articles sur le site : www.edpsciences.org/meca 